\title{
Hypoglycemic Effect of Aqueous Shallot and Garlic Extracts in Rats with Fructose-Induced Insulin Resistance
}

\author{
Razieh Jalal ${ }^{1 *}$, Sayyed Majid Bagheri ${ }^{2}$, Ali Moghimi ${ }^{2}$, and Morteza Behnam Rasuli ${ }^{2}$ \\ ${ }^{1}$ Department of Chemistry, Ferdowsi University of Mashhad, Mashhad 91779, Iran \\ ${ }^{2}$ Department of Biology, Ferdowsi University of Mashhad, Mashhad 91779, Iran
}

Received 9 April 2007; Accepted 11 May, 2007

\begin{abstract}
Summary The present study has been carried out to investigate the effect of aqueous extract of shallot (Allium ascalonicum) and garlic (Allium satium) on the fasting insulin resistance index (FIRI) and intraperitoneal glucose tolerance test (IPGTT) of fructose-induced insulin resistance rats. Male albino Wistar rats were fed either normal or high-fructose diet for a period of eight weeks. Fasting blood glucose level, fasting blood triglyceride level, FIRI, and the area under the glucose tolerance curve were significantly elevated in fructose-fed animals. Fructose-induced insulin resistance rats treated by aqueous shallot or garlic extract $(500 \mathrm{mg} /$ kg body weight/day, i.p.) for duration of eight weeks. Control animals only received normal saline $(0.9 \%)$. The results showed that neither shallot nor garlic extracts significantly altered the FIRI and the IPGTT at the fourth week after treatment. The fasting blood glucose in fructose-induced insulin resistance animals has been significantly decreased in 8-week treated animals by both shallot and garlic extracts. Shallot extract administration, but not garlic extract, for a period of eight weeks can significantly improve the intraperitoneal glucose tolerance and diminish the FIRI. These results indicate that shallot and garlic extracts have a hypoglycemic influence on the fructose-induced insulin resistance animals and aqueous shallot extract is a stronger hypoglycemic agent than the garlic extract.
\end{abstract}

Key Words: insulin resistance, garlic, shallot, glucose tolerance

\section{Introduction}

The public health burden of type 2 diabetes mellitus (T2DM) has been dramatically increased worldwide. It has been shown that the risk for developing clinical diabetes is substantially increased in the state of impaired fasting glycemia or impaired glucose tolerance. Fasting hyperglycemia is caused by unrestrained basal hepatic glucose output, primarily a consequence of hepatic resistance to insulin action [1]. Insulin resistance not only plays an important role in

*To whom correspondence should be addressed.

Tel: +98-511-8797022 (Ext. 311) Fax: +98-511-8796416

E-mail: razieh@ferdowsi.um.ac.ir (rsjalal@yahoo.com)
T2DM but it also is an extremely common feature of a number of important human diseases including atherosclerosis, hypertension, and dyslipidemia [2-5].

Many traditional plants have long been used effectively in treating many diseases in the world. It has been shown garlic belongs to the Liliaceae family and has diverse biological activities $[6,7]$. The number of studies pointed to the hypoglycemic effect of garlic is limited; however, the results of such studies are inconsistent. Jain and Vyas [8] have been shown the hypoglycemic effect of garlic extracts with water or several other different organic solvents on the oral glucose tolerance in both normal and alloxan-induced diabetic rabbits. The hypoglycemic effect of garlic oil in diabetic animals and humans has also been reported [9-12]. However, some researchers failed to show the hypoglycemic 
effect of garlic in streptozotocin-induced diabetic animal models [13, 14]. About the cause of these inconsistent results, some ideas have been suggested but the exact reason is unclear.

Shallot that belongs to the Liliaceae family is a major component of many Asian diets and is widely believed to be beneficial for health. This bulb is darker than garlic and has a stronger odor that correlates with its sulfide component [15]. As far as we searched, there is no previous trial study showing the effects of shallot on glycemic control in insulin resistance animal models. The aim of the present study is to compare the influence of persian shallot aqueous extract with the garlic extract on the FIRI and IPGTT in insulin resistance rats.

\section{Materials and Methods}

\section{Preparation of shallot and garlic extracts}

Fresh shallot (Allium ascalonicum) and garlic (Allium satium) bulbs were obtained from the local market in Mashhad, Iran. The shallot and/or garlic bulbs (150 g) in good physical shapes were peeled and homogenized in $100 \mathrm{ml}$ of cold, sterile $0.9 \%$ saline in the presence of crushed ice. The homogenization was carried out in a high speed blender for $15 \mathrm{~min}$. The homogenized mixture was filtered 3 times through cheesecloth. The volume of the resulting aqueous extracts was made up to $200 \mathrm{ml}$ with $0.9 \%$ saline. The concentrations of the shallot and garlic extracts were considered to be $750 \mathrm{mg} / \mathrm{ml}$. The prepared extracts were stored at $-20^{\circ} \mathrm{C}$ until use. Shallot and garlic extracts of lower concentrations were prepared by dilution of these solutions with cold sterile $0.9 \%$ saline.

\section{Animals and treatments}

Thirty four male albino Wistar rats weighing 180-240 g were purchased from the animal house of Razi research center in Mashhad, Iran. Animal handling was performed with regard to Iranian animal ethics society and local university rules. The animals were conditioned at room temperature and natural photoperiods. After two weeks of acclimation, the animals were divided into two groups. Each group of rats was separately housed in standard cages and had free access to water and standard pellet diet. The first group (10 rats) was used as control and fed with standard rat chow and tap water. Insulin resistance was induced by fructose administration. To produce the insulin resistance animals, the second group (24 rats) received normal chow such as control group and $10 \% \mathrm{w} / \mathrm{v}$ fructose dissolved in drinking water for a period of eight weeks $[16,17]$. Eight weeks later the fasting blood glucose, blood triglyceride, and serum insulin levels were determined in both groups. The fructose-fed rats were considered as insulin resistance animals. Following induction of insulin resistance in the second group, they were further divided into three subgroups $(n=8)$. The first subgroup was kept as insulin resistance (sham control) while the second and third subgroups were daily treated by injection (i.p.) $500 \mathrm{mg} / \mathrm{kg}$ body weight (BW) of shallot and garlic extract, respectively, for a period of eight weeks. The control and sham groups received $500 \mathrm{ml}$ of $0.9 \%$ saline in the same manner.

\section{Biochemical Assays}

Blood glucose, blood triglyceride, and serum insulin levels were measured before and after fructose-feeding, and at 4th and 8th weeks of shallot and/or garlic extract treatment. For this purpose, blood samples were collected from retro-orbital plexus using heparinized microhematocrit tubes. Blood glucose and triglyceride levels were immediately determined by a standard glucose/triglyceride/cholesterol meter (Accutrend GCT, Roche, Germany). In order to prepare serum, the blood was collected in a centrifuge tube and allowed clotting. After centrifuging at $1500 \mathrm{rpm}$ for $10 \mathrm{~min}$, the supernatant (serum) was collected and the level of serum insulin was estimated by the immunoradiometric assay kit (Immunotech, Marseille, France).

\section{Insulin resistance index calculation}

Fasting insulin resistance index (FIRI) was calculated according to the formula [18]:

$$
\text { FIRI }=\frac{\text { Fasting insulin }(\mathrm{mU} / \mathrm{ml}) \times \text { Fasting glucose }(\mathrm{mg} / \mathrm{dl})}{25}
$$

\section{Intraperitoneal glucose tolerance test (IPGTT)}

Intraperitoneal glucose tolerance test was performed by intraperitoneal injection of a $40 \%$ glucose solution $(2 \mathrm{~g} / \mathrm{kg}$ BW). Blood samples were collected from retro-orbital plexus using heparinized microhematocrit tubes immediately before (zero time) and 30, 60, and $120 \mathrm{~min}$ after the glucose loading. Blood glucose level was measured with a standard glucose/triglyceride/cholesterol meter (Accutrend GCT, Roche, Germany) and expressed as milligrams per deciliter.

\section{Statistical data analysis}

Data are expressed in terms of mean \pm standard deviation (SD). Differences in blood glucose, blood triglyceride, and FIRI between control group and insulin resistance group were analyzed by the Student's $t$ test. Duncan's multiplecomparison test was used to compare the mean values of fasting blood glucose, FIRI, and the area under the glucose tolerance curve of three fructose-fed subgroups. $p$ values less than 0.05 were considered statistically significant. 


\section{Results}

Induction of insulin resistance with fructose was associated with increasing body weight, fasting blood glucose level, fasting blood triglyceride concentration, and fasting insulin resistance index (Table 1). The body weight and blood glucose level were higher in the insulin resistance animals

Table 1. Body weight, blood glucose level, fasting insulin resistance index, and blood triglyceride level of fructosefed and control groups after induction of insulin resistance.

\begin{tabular}{lcc}
\hline & Control* & Insulin Resistance \\
\hline Body weight $(\mathrm{g})$ & $245.23 \pm 17.87$ & $265.13 \pm 17.72$ \\
Glucose $(\mathrm{mg} / \mathrm{dl})$ & $140.21 \pm 15.54$ & $152.05 \pm 17.76$ \\
FIRI & $19.92 \pm 5.30$ & $23.97 \pm 4.38$ \\
Triglyceride $(\mathrm{mg} / \mathrm{dl})$ & $73.47 \pm 5.75$ & $105.09 \pm 25.93$ \\
\hline
\end{tabular}

Values are the mean \pm SD for at least 10 rats per group. ${ }^{*} p<0.05$ (compared to the insulin resistance group).
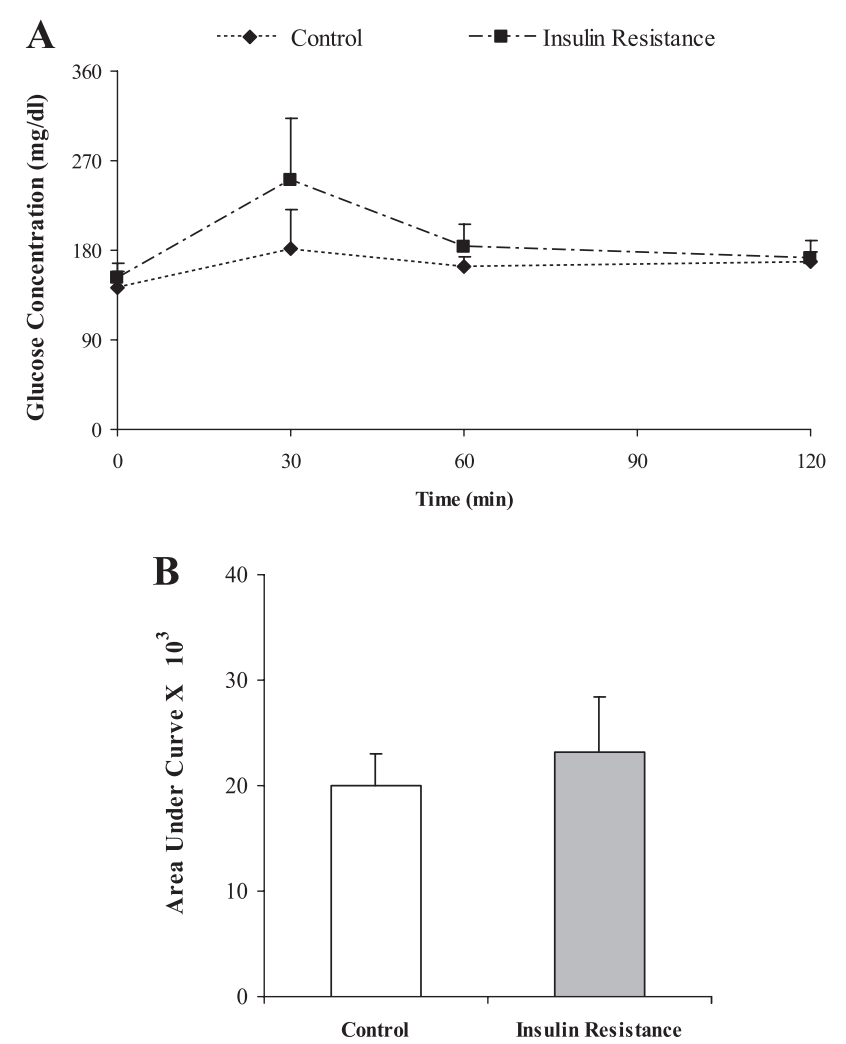

Fig. 1. The effect of fructose-feeding on the intraperioneal glucose tolerance curve. Glucose solution $(2 \mathrm{~g} / \mathrm{kg}$ body weight) was administered intraperitoneally, blood glucose concentrations were measured 30, 60, $120 \mathrm{~min}$ afterward. Fasting blood glucose is expressed as values for zero time. The data in (B) are calculated from the areas under the curves of (A). Data are the mean $\pm \mathrm{SD}$ of at least 10 rats per group. $p<0.05$ (compared to the insulin resistance group). than in the controls. Compared to the control group, the FIRI and the fasting blood triglyceride concentrations were significantly increased in the fructose-fed rats $(p<0.05)$. After intraperitoneal glucose loading, the blood glucose concentration in fructose-fed rats was increased dramatically compared to the control animals. Also, the area under the glucose tolerance curve was significantly greater in the fructose-fed group than in the control group (Fig. 1) $(p<0.05)$.

The effects of the garlic and shallot aqueous extracts on the intraperitoneal glucose tolerance were studied at 4 th and 8 th weeks of the insulin resistance induction. At the 4 th week, the blood glucose concentration profile as a function of the time during the intraperitoneal glucose tolerance test for the insulin resistance animals with shallot or garlic aqueous extracts treatment were almost similar to those of the control and sham rats (Fig. 2A). As shown in Fig. 2B,
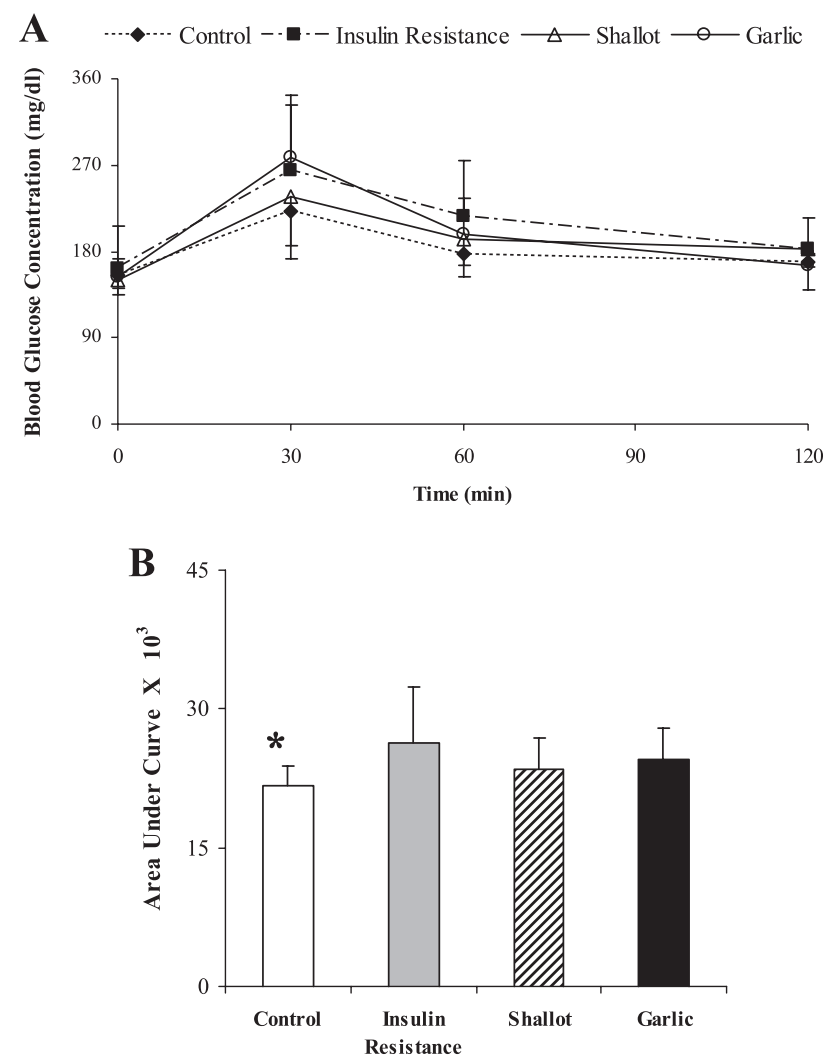

Fig. 2. The effect of treatment of insulin resistance rats with shallot or garlic aqueous extract on the intraperitoneal glucose tolerance during the 4-week treatment period. Glucose solution ( $2 \mathrm{~g} / \mathrm{kg}$ body weight) was administered intraperitoneally, blood glucose concentrations were measured 30, 60, and 120 min afterward. Fasting blood glucose is expressed as values for zero time. The data in (B) are calculated from the areas under the curves of (A). Data are the mean $\pm \mathrm{SD}$. ${ }^{*} p<0.05$ (compared to the insulin resistance group). 
treatment with shallot extract slightly reduced the area under the glucose tolerance curve. Eight weeks treatment with shallot aqueous extract could improve intraperitoneal glucose tolerance and significantly decrease the area under the glucose tolerance curve in insulin resistance rats $(p<0.05)$, whereas garlic aqueous extract could slightly diminish the area under the glucose tolerance curve (Fig. 3).

After eight weeks administration, fasting blood glucose level in the shallot and garlic groups was significantly lower than in the sham (Table 2) $(p<0.05)$. FIRI of different groups are shown in Table 3. FIRI was significantly decreased by shallot extract $(22.80 \pm 6.10)$ when compared with the sham $(34.54 \pm 11.38)$ at eight weeks after the treatment $(p<0.05)$.
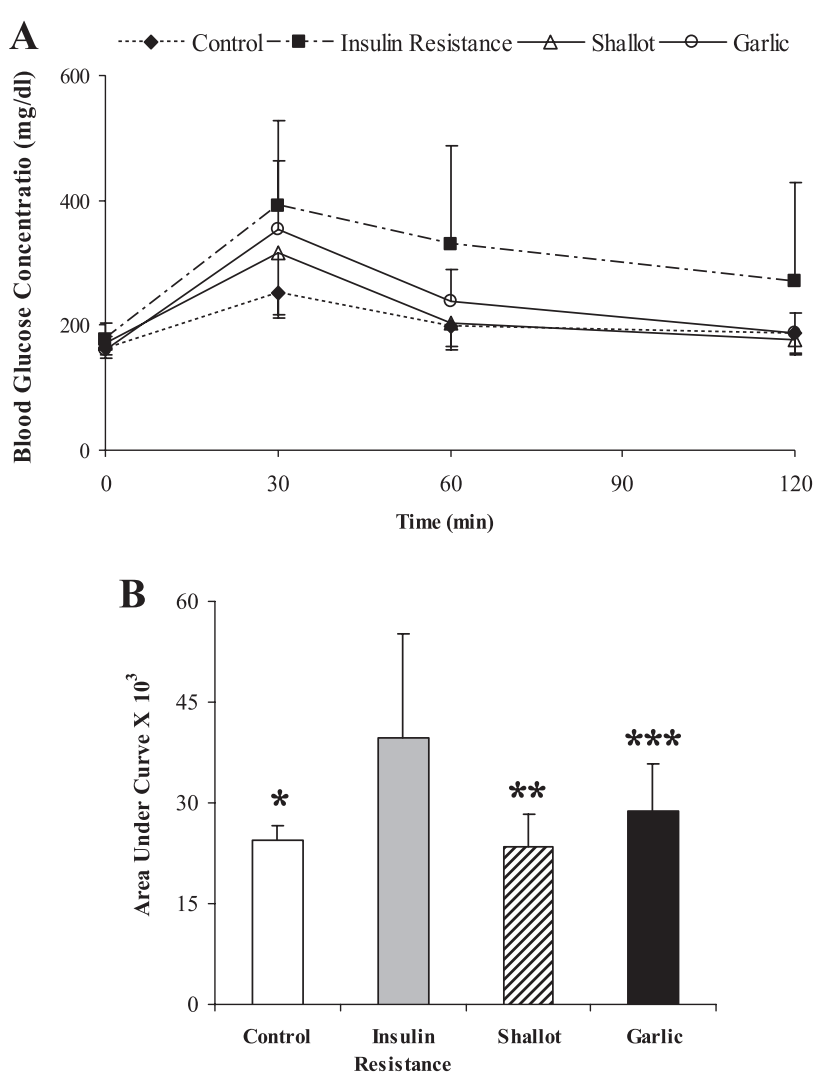

Fig. 3. The effect of treatment of insulin resistance rats with shallot or garlic aqueous extract on the intraperitoneal glucose tolerance during the 8 -week treatment period. Glucose solution ( $2 \mathrm{~g} / \mathrm{kg}$ body weight) was administered intraperitoneally, blood glucose concentrations were measured 30, 60, and 120 min afterward. Fasting blood glucose is expressed as values for zero time. The data in (B) are calculated from the areas under the curves of (A). Data are the mean \pm SD. ${ }^{*} p<0.05,{ }^{* *} p<0.05,{ }^{* * *} p<0.05$ (significantly difference from the insulin resistance group).

\section{Discussion}

It is believed that poorly controlled blood glucose is the most important factor in the development of diabetes. Insulin resistance is considered as a part of the metabolic risk profile including central obesity, cardiovascular, hypertension, and diabetes. Previous studies have shown that long-term fructosefeeding induces mild insulin resistance in experimental animals [19-21]. In the present study, rats were fed with a high-fructose diet for a period of eight weeks in order to induce insulin resistance. As indicated in the previous studies on rats and other rodents (e.g. hamster) [20], the results of the present study show that fructose-feeding induces hypertriglyceridemia (Table 1). This is believed to be related to the hypertriglyceridemic effect of fructose [22]. Fructose feeding stimulates the hepatic production of triglycerides, both by promoting the reestrification of circulating non-esterified fatty acids and also by stimulating de novo fatty acid synthesis [23]. Increased delivery of triglycerides or non-esterified fatty acids to the muscle interferes with the utilization of glucose, through the principles of Randle cycle [24], and also impairs the insulin action. Our results show that fructose-fed group has a higher blood glucose level, FIRI, and blood triglyceride level than that of control group. The measured area under the glucose tolerance curve was significantly greater in insulin resistance group compared to the control group throughout the investigation period. Thus, the fructose-fed animals displayed characteristics typical of insulin resistance.

Table 2. The effect of treatment of insulin resistance rats with shallot or garlic aqueous extract on the fasting blood glucose level at 8-week treatment period.

\begin{tabular}{lc}
\hline Animal groups & Glucose $(\mathrm{mg} / \mathrm{dl})$ \\
\hline Control $(n=10)^{*}$ & $166.92 \pm 14.26$ \\
Insulin Resistance $(n=6)$ & $194.13 \pm 27.63$ \\
Shallot $(n=6) * *$ & $147.14 \pm 36.37$ \\
Garlic $(n=8)^{* * *}$ & $156.5 \pm 15.38$
\end{tabular}

Data are the mean \pm SD. ${ }^{*} p<0.05, * * p<0.05, * * * p<0.05$ (significantly difference from the insulin resistance group).

Table 3. The effect of treatment of insulin resistance rats with shallot or garlic aqueous extract on the fasting insulin resistance index (FIRI) at 8-week treatment period.

\begin{tabular}{ll}
\hline Animal groups & \multicolumn{1}{c}{ FIRI } \\
\hline Control $(n=10)^{*}$ & $19.51 \pm 5.17$ \\
Insulin Resistance $(n=6)$ & $34.54 \pm 11.38$ \\
Shallot $(n=6)^{* *}$ & $22.80 \pm 6.10$ \\
Garlic $(n=8)$ & $27.08 \pm 9.89$
\end{tabular}

Data are the mean \pm SD. $* p<0.05, * * p<0.05$ (significantly difference from the insulin resistance group). 
Garlic and shallot are widely consumed as components of the diet and also as a traditional medication in many countries. Extensive studies have carried out on garlic extracts. They reported the presence of flavonoids and sulfur-containing compounds [25, 26]. Garlic and garlic constituents have been shown to have diverse biological activities, including anticarcinogenic, antithrombotic, antimicrobial, antioxidant, antidabetic, and various other biological actions $[6,7,27]$. There are controversial and opposite results observed in different researches using garlic preparations on blood glucose level. Jain et al. $[8,28]$ investigated the effect of extracts of garlic with water or several different organic solvents on oral glucose tolerance in normal and alloxaninduced diabetic rabbits. They found that all kind of garlic preparations possesses an acute hypoglycemic effect. Recently, it is reported that garlic juice reverse hyperglycemic in alloxaninduced diabetic rats [29]. However, Baluchnejadmojarad and Rohgani found no hypoglycemic effect of an aqueous extract of garlic in streptozotocin (STZ)-induced diabetic rats [13]. In the present study, the effects of garlic extract on glucose tolerance and FIRI in fructose-induced insulin resistance animals has been studied. Treatment of fructoseinduced insulin resistance rats with aqueous garlic extract (500 mg/kg BW/day, i.p.) for a period of four weeks did not have any effect on the intraperitoneal glucose tolerance. At the 8th weeks of treatment, the area under the glucose tolerance curve, the fasting blood glucose, and the FIRI were decreased by $13,19.3$, and $21.6 \%$, respectively, relative to the insulin resistance rats.

Although numerous scientific studies have been undertaken to test the basis and validity of garlic preventive and curative potential against a range of diseases, little parallel work has been carried out with shallot. Analysis of shallot extracts has confirmed the presence of falvonols such as quercetin, and sulfur compounds such as diallyl disulfide [30,31]. Current knowledge of the properties and constituents of shallot and its analogy with garlic suggests that some biological activities of shallot extracts may be similar to garlic extracts. The effect of shallot extract on the glucose tolerance and insulin resistance index has not been investigated before. Our results show that in vivo administration of shallot extract for a period of eight weeks, but not 4-week, could improve glucose tolerance (32\%) and decrease fasting blood glucose $(24.2 \%)$ and FIRI (34\%) in insulin resistance rats.

Like many chronic disease, chronic hyperglycemia is widely believed to cause elevated concentrations of reactive oxygen species accompanied by lowered enzymatic and nonenzymatic cell antioxidant defenses [32, 33]. Reactive oxygen species have been suggested to be involved in beta cell dysfunction and insulin resistance [34]. The beneficial effect of shallot and garlic extracts on insulin resistance state may be due to the antioxidant capacity of these compounds. Leelarungrayub et al. showed that organic solvent and aqueous extracts of garlic and shallot bulbs had significant antioxidant potential, as measured by decrease in free radicals and ability to inhibit lipid oxidation. They showed that shallot extracts have antioxidant properties similar to or slightly higher than those of garlic extracts. The relative antioxidant potentials of the garlic and shallot extracts were related to their content of phenolic and sulfur compounds [31]. Previous studies reported that the total content of phenolic and diallyl disulfide compounds in shallot extract were higher than in garlic $[35,36,37]$.

In conclusion, the results of the present study indicate that neither shallot nor garlic extracts had a significant effect on the FIRI and the glucose tolerance at the 4th week of treatment, but both shallot and garlic extracts can significantly decrease fasting blood glucose in insulin resistance rats at the 8th week post-treatment. The IPGTT and the FIRI were significantly improved only after 8-weeks treatment with shallot extract. These results suggest that aqueous shallot extract is a stronger hypoglycemic agent than the garlic extract and it may be a useful supplemental remedy in insulin resistance state. However, further studies should be performed to find out the effectiveness of long-term treatment and the best effective dose of these plants in fructose-fed animal model of insulin resistance.

\section{Acknowledgments}

This study was supported by grants from Ferdowsi University of Mashhad. The authors gratefully acknowledge Dr. M. Ejtehadi and the excellent technical assistance of Dr. Ejtehadi Medical Lab. Center, S. Vafadar.

\section{References}

[1] Daily, G.: New strategies for basal insulin treatment in type 2 diabetes mellitus. Clin. Ther., 6, 889-901, 2004.

[2] Adeli, K., Taghibiglou, C., Van Iderstine, S.C., and Lewis, G.F.: Mechanisms of hepatic very low-density lipoprotein overproduction in insulin resistance. Trends Cardiovasc. Med., 11, 170-176, 2001.

[3] Moller, D.E. and Flier, J.S.: Insulin resistance-mechanisms, syndromes, and implications. N. Engl. J. Med., 325, 938948, 1991.

[4] Reaven, G.M.: Banting lecture role of insulin resistance in human disease. Diabetes, 37, 1595-1607, 1988.

[5] Shiota, G. and Tsuchiya, H.: Pathophysiology of NASH: Insulin resistance, free fatty acids and oxidative stress. J. Clin. Biochem. and Nutr., 38, 127-132, 2006.

[6] Agarwal, K.C.: Therapeutic actions of garlic constituents. Med. Res. Rev., 16, 111-124, 1996.

[7] Augusti, K.T.: Therapeutic values of onion (Allium cepa L.) and garlic (Allium sativum L.). Indian J. Exp. Biol., 34, 634640, 1996.

[8] Jain, R.C. and Vyas, S.R.: Garlic in alloxan-induced diabetic 
rabbits. Am. J. Clin. Nutr., 28, 684-685, 1975.

[9] Anwar, M.M. and Meki, A.R.: Oxidative stress in streptozotocininduced diabetic rats: effects of garlic oil and melatonin. Comp. Biochem. Physiol., Part A Mol. Integr. Physiol., 135, 539-547, 2003.

[10] Duncan, M.G.: The effects of nutritional supplements on the treatment of depression, diabetes, and hypercholesterolemia in the renal patient. J. Ren. Nutr., 9, 58-62, 1999.

[11] Liu, C.T., Hse, H., Lii, C.K., Chen, P.S., and Sheen, L.Y.: Effects of garlic oil and diallyl trisulfide on glycemic control in diabetic rats. Eur. J. Pharmacol., 516, 165-173, 2005.

[12] Liu, C.T., Wong, P.L., Lii, C.K., Hse, H., and Sheen, L.Y.: Antidiabetic effect of garlic oil but not diallyl disulfide in rats with streptozotocin-induced diabetes. Food. Chem. Toxicol., 44, 1377-1384, 2006.

[13] Baluchnejadmojarad, T. and Roghani, M.: Garlic extract attenuates time-dependent changes in the reactivity of isolated aorta in streptozotocin-diabetic rats. Life Sci., 73, 22812289, 2003.

[14] Swanston-Flatt, S.K., Day, C., Bailey, C.J., and Flatt, P.R.: Traditional plant treatments for diabetes. Studies in normal and streptozotocin diabetic mice. Diabetologia, 33, 462-464, 1990.

[15] Mubarak, A.M. and Kulatilleke, C.P.: Sulfur constituents of need seed volatiles: a revision. Phytochemistry, 29, 33513352, 1990.

[16] Elliott, S.S., Keim, N.L., Stern, J.S., Teff, K., and Havel, P.J.: Fructose, weight gain, and the insulin resistance syndrome. Am. J. Clin. Nutr., 76, 911-922, 2002.

[17] Hwang, J.-S., Ho, H., Hoffman, B.B., and Reaven, G.M.: Fructose- induced insulin resistance and hypertension in rats. Hypertension, 10, 512-516, 1987.

[18] Duncan, M.H., Singh, B.M., Wise, P.H., Carter, G., and Alaghband-Zadeh, J.: A simple measure of insulin resistance. Lancet, 346, 120-121, 1995.

[19] Basciano, H., Federico, L., and Adeli, K.: Fructose, insulin resistance, and metabolic dyslipidemia. Nutr Metab (Lond), 2, 5, 2005.

[20] Bezerra, R.M., Ueno, M., Silva, M.S., Tavares, D.Q., Carvalho, C.R., Saad, M.J., and Gontijo, J.A.: A high-fructose diet induces insulin resistance but not blood pressure changes in normotensive rats. Braz. J. Med. Biol. Res., 34, 1155-1160, 2001.

[21] D’Angelo, G., Elmarakby, A.A., Pollock, D.M., and Stepp, D.W.: Fructose feeding increases insulin resistance but not blood pressure in Sprague-Dawley rats. Hypertension, 46, 806-811, 2005.

[22] Lee, M.K., Miles, P.D., Khoursheed, M., Gao, K.M., Moossa, A.R., and Olefsky, J.M.: Metabolic effects of troglitazone on fructose-induced insulin resistance in the rat. Diabetes, $\mathbf{4 3}$, 1435-1439, 1994.

[23] Southgate, D.A.: Digestion and metabolism of sugars. Am. J.
Clin. Nutr., 62, 203S-211S, 1995.

[24] Randle, P.J.: Regulatory interactions between lipids and carbohydrates: the glucose fatty acid cycle after 35 years. Diabetes Metab. Rev., 14, 263-283, 1998.

[25] Kim, S.M., Kubota, K., and Kobayashi, A.: Antioxidative activity of sulfur containing flavor compounds in garlic. Biosci. Biotechnol. Biochem., 61, 1482-1485, 1997.

[26] Kodera, Y., Suzuki, A., Imada, O., Kasuga, S., Sumioka, I., Kanezawa, A., Taru, N., Fujikawa, M., Nagae, S., Masamoto, K., Maeshige, K., and Ono, K.: Chemical, and biological properties of s-allylcysteine, an amino acid derived from garlic. J. Agric. Food Chem., 50, 622-632, 2002.

[27] Azuma, K., Minami, Y., Ippoushi, K., and Terao, J.: Lowering effects of onion intake on oxidative stress biomarkers in streptozotocin-induced diabetic rats. J. Clin. Biochem. Nutr., 40, 131-140, 2007.

[28] Jain, R.C., Vyas, C.R., and Mahatma, O.P.: Letter: Hypoglycaemic action of onion and garlic. Lancet, 2, 1491, 1973.

[29] El-Demerdash, F.M., Yousef, M.I., and El-Naga, N.I.: Biochemical study on the hypoglycemic effects of onion and garlic in alloxan-induced diabetic rats. Food Chem. Toxicol., 43, 57-63, 2005.

[30] Fattorusso, E., Iorizzi, M., Lanzotti, V., and Taglialatela-Scafati, O.: Chemical composition of shallot (Allium ascalonicum Hort.). J. Agric. Food Chem., 50, 5686-5690, 2002.

[31] Leelarungrayub, N., Rattanapanone, V., Chanarat, N., and Gebicki, J.M.: Quantitative evaluation of the antioxidant properties of garlic and shallot preparations. Nutrition, 22, 266-274, 2006.

[32] Bonnefont-Rousselot, D., Bastard, J.P., Jaudon, M.C., and Delattre, J.: Consequences of the diabetic status on the oxidant/ antioxidant balance. Diabetes Metab., 26, 163-176, 2000.

[33] Catherwood, M.A., Powell, L.A., Anderson, P., McMaster, D., Sharpe, P.C., and Trimble, E.R.: Glucose-induced oxidative stress in mesangial cells. Kidney Int., 61, 599-608, 2002.

[34] Evans, J.L., Goldfine, I.D., Maddux, B.A., and Grodsky, G.M.: Are oxidative stress-activated signaling pathways mediators of insulin resistance and $\beta$-cell dysfunction? Diabetes, 52, 1-8, 2003.

[35] Leelarungrayub, N., Chanarat, N., and Rattanapanone, V.: Potential activity of Thai shallot (Allium Ascalonicum L.) extract on the prevention of hemolysis and glutathione depletion in human erythrocyte from oxidative stress. $C M U$ J., 3, 225-234, 2004.

[36] Terrance, L., Charles, G., Larry, F., William, K.H., Jose C., and Vicente N.: Molecular Characterization of quercetin and quercetin glycosides in Allium vegetables. Their effects on malignant cell transformation. ACS Symp. Ser., 50, 220-238, 1992.

[37] Benzanger-Beauquesne, L., and Delelis, A.: Flavonoids from Allium ascalonicum bulbs. Compt Rend Ser D Sci Nat., 265, 2118-2119, 1967. 